\title{
Implementation and performance analysis of a new family of coherent 3- dimensional space/phase/frequency spread optical code for OCDMA networks
}

\author{
Himanshu Monga, Dr R.S. Kaler
}

Department of Electronics and Communication Engg, Thapar University, Patiala (Punjab)-INDIA

\section{Council for Innovative Research}

Peer Review Research Publishing System

Journal of Social Science Research

Vol.3, No.2

editor@jssronline.com

www.cirworld.com, www.jssronline.com

\section{ABSTRACT}

In his paper, we have demonstrated the feasibility of a spectrally efficient wavelength-division-multiplexing-compatible optical code-division multiple-access system using 16 phase-locked laser lines within a 40-GHz tunable window as frequency and an ultrahigh frequency resolution spectral phase encoder-decoder. Coding and decoding slots using binary $[0, \pi]$ phase chips were demonstrated for four users at $5 \mathrm{~Gb} / \mathrm{s}$, and a single coded signal was separated from four copropagating signals, with bit-error rate $<10-9$.

\section{Index Terms:}

Code-division multiple-access (CDMA); code division multiplexing; optical code-division multiple-access (OCDMA),; optical communications; wavelength-division multiplexing (WDM).

\section{I.INTRODUCTION}

Optical code-division multiple-access (O-CDMA) is an attractive technology since it potentially provides flexible, robust, and asynchronous communications in access networks.CDMA schemes are categorized as implementing the code through the optical field and relying on coherent detection, or through time slots and wavelengths with reliance on incoherent detection. Coherent schemes are susceptible to coherent beat noise that occurs when the correctly decoded signal temporally overlaps with the multiple access interference (MAI) from other users [1].So recent implementations of coherent O-CDMA resort to timing coordination between users, ranging from complete bit-level synchronization [2] to time slot assignment within the bit [3]. Another approach uses very long spreading codes to minimize the amplitude of the MAI while keeping users asynchronous; however, only ten of 512 possible codes deliver adequate bit-error-rate performance $(B E R<10-9)[4]$. In-coherent schemes are less susceptible to coherent interference [5], but due to time slot allocation, are difficult to implement and less spectrally efficient with increasing data rates and time slots.

Optical code division multiple access has recently been of immense interest for its ability to support asynchronous and bursty traffic, its security against interception and good performance under multiple access conditions. For unipolar signals, the length of the one-dimensional sequences becomes very large in order to accommodate a large number of simultaneous users with sufficient orthogonality among the codes [6].

To overcome this drawback, 2-D codes were developed, where the optical pulses are spread in wavelength-time domain [7] or in space-time domain [8].

R.S.Kaler et al [9] demonstrated the influence of EDFA power and increase in length of each type of fiber on the performance of optical communication systems also observed that system needs proper matching between the EDFA power and length of the fiber for optimum performance.

We have reported a 3D Optical CDMA system with increased data rate i.e. $5 \mathrm{~Gb} / \mathrm{s}$ and even with increased number of users and achieved a spectral efficiency of $12.5 \%(6 * 5 \mathrm{~Gb} / \mathrm{s}$ in an $40-\mathrm{GHz}$ window). Spectrum spreading is further limited by the preferred use of single-sideband modulation over on-off key modulation. Results obtained in terms of eye-diagram \& BER indicate that for six simultaneous users transmitting at $5 \mathrm{~Gb} / \mathrm{s}$ and using a suitably chosen set of codes among the 
set 16 Hadamard codes of length 16 , up to 12 ps of relative delay can be tolerated with a power penalty within $1 \mathrm{~dB}$ at a bit-error rate $(B E R)$ of $\leq 10-9$.

(a)

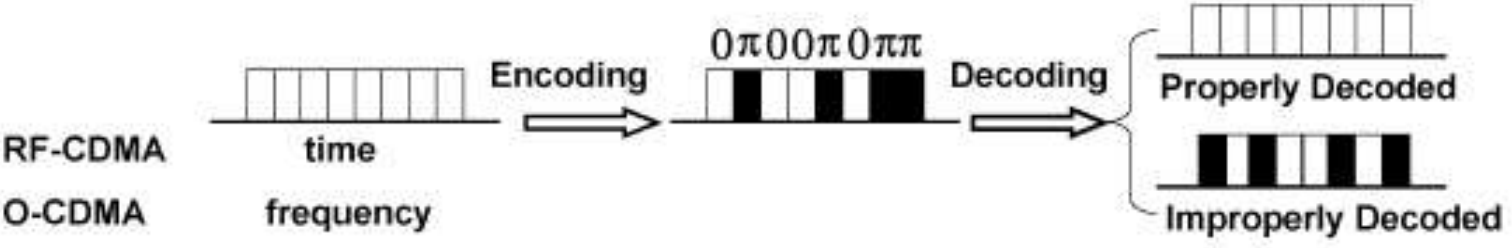

(b)

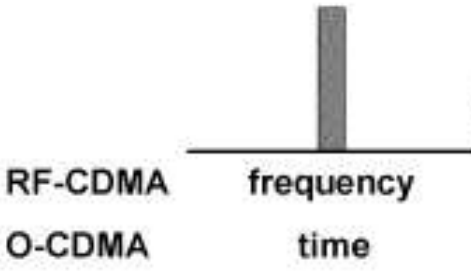

同

Properly Decoded

Improperly Decoded

Fig.: 1.1 Comparison of DS RF-CDMA and spectral-phase-coded OCDMA [10]. (a) Time-domain behavior for RF-CDMA (equivalent to frequency-domain behavior for OCDMA). (b) Frequency-domain behavior for RFCDMA (equivalent to time-domain behavior for O-CDMA).

The proposed ultra-short-pulse OCDMA scheme based on spectral-phase- frequency encoding has an interesting analogy with the most widely used direct sequence (DS) RF-CDMA. Fig. 1.1 above shows time- and frequencydomain evolution during encoding/decoding for these two schemes. For DS RF-CDMA, a bit is divided into many temporal chips and phase-coded for each chip [for example, 0 or phase shift for bipolar phase shift keying (BPSK)] in the time domain [Fig. 2(a)] so that the spectrum of a narrow-band signal is broadened during the encoding process [Fig. 2(b)]. After decoding at the receiver, a properly decoded signal is recovered back to a narrow-band signal, which can be separated out by a narrow-band filter from the improperly decoded broad-band MAI. The proposed OCDMA scheme is just a timefrequency-reversed version of DS RF-CDMA, where the ultra-short-pulse spectrum is divided to many frequency chips and phase-coded for each chip [Fig. 2(a)]. This results in spreading in the time domain [Fig. 2(b)], while proper decoding "despreads" the encoded signal back to its original duration in time.

The paper is organized in to five sections, after introduction in section I, section II gives brief introduction of coding concept \& its generation, section III provides the simulation setup of optical CDMA system employing spectralphase coding in time domain. Results are compared and discussed in Section IV, followed by conclusion in section $\mathrm{V} \&$ finally references are given in section VI.

\section{Coding Concept \& Generation}

The encoding process begins with a train of short pulses, with spectral content comprising a stable comb of closely spaced phase-locked frequencies; these are generated by an MLL/PLL and have a frequency spacing equal to the MLL/PLL pulse repetition rate. Here number of wavelengths is divided among different channels accordingly the number of time slots or delays are given, and also converting the electrical signal into optical signal. Hence at the transmitter end, spreading in the space domain takes place.

Actual encoding consists of separating each of these frequency slots, shifting its phase, in this case by 0 or $\pi$ as prescribed by the choice of code, and recombining the frequency bins to produce the coded signal. When the relative phases of the frequencies are shifted, the set of frequencies are unaltered, but their recombination results in a different temporal pattern: e.g., a pulse shifted to a different part of the bit period, multiple pulses within the bit period. Each OCDMA code is defined by a unique choice of phase shifts according to choice of codes

Here, in the proposed system we have chosen the set of hadamard codes which are by nature orthogonal and binary. The generated codes are then converted to phase codes by assigning to -1's and 1's phase shifts of ' 0 ' and '. 6 simultaneous users transmitting at $5 \mathrm{~Gb} / \mathrm{s}$ employing hadamard codes of length 16 is used in this system. This choice of hadmard codes is based on the goal of high spectral efficiency with minimal multiuser interference (MUI). Unlike many optical coding schemes that have been proposed, ours offers true orthogonally in the sense that MUI is zero at the time that the decoded signal is maximum. The number of orthogonal codes is equal to the number frequency bins; hence, high spectral efficiency is possible.

A Z-element Hadamard code is a row from $Z \times Z$ orthogonal Hadamard matrix, which has $(1,-1)$ valued binary entries. The $Z \times Z$ Hadamard matrix $H M$ where $Z=2 M[16]$ is generated by the code matrix:

$$
H_{M}=\left[\begin{array}{ll}
1 & 1 \\
1 & -1
\end{array}\right]
$$

For $\mathrm{M}=2$, the Hadamard matrix is generated as below [16]: 


$$
H_{2}=\left[\begin{array}{ll}
H_{1} & H_{1} \\
H_{1} & \tilde{H}_{1}
\end{array}\right]=\left[\begin{array}{llll}
1 & 1 & 1 & 1 \\
1 & -1 & 1 & -1 \\
1 & 1 & -1 & -1 \\
1 & -1 & -1 & 1
\end{array}\right]
$$

Unipolar Hadamard matrix HM having the following properties

1. M should be greater than or equal to 2 .

2. Code length $\mathrm{N}=2 \mathrm{M}$.

3. Code weight $\mathrm{w}=2 \mathrm{M}-1$.

4. User $\mathrm{K}=2 \mathrm{M}-1$ (the case $\mathrm{Z}=1$ has been excluded since the row of the unipolar Hadamard matrix is all ones).

5 The ratio of $W / \lambda=2$ ( $\lambda$ is cross-correlation properties).

$A(Z \times Z)$ Hadamard matrix of 1 's and -1 's has the property that any row differs from any other row in exactly $Z / 2$ positions. All rows except one contains $Z / 2(-1)$ 's and $Z / 2$ (1)'s, usually in wavelength domain we replace the -1 with 0 . The sequence $(1,0)$ is a unipolar Hadamard code for example, for $Z=4$.

$$
H_{2}=\begin{array}{llll}
1 & 1 & 1 & 1 \\
1 & 0 & 1 & 0 \\
1 & 1 & 0 & 0 \\
1 & 0 & 0 & 1
\end{array}
$$

This code can support $2 \mathrm{M}-1$ number of users.

For example, if only 20 users are required, $M$ will have to be at least equivalent to 5 which supports up to 31 users, thus rendering 11 codes as unused. It is clear that Walsh Hadamard code is not efficient code because the sequence cannot be constructed exactly according to the number of users. It has increasing value of cross-correlation as the number of user increases. And it also requires more number of filters for each code as the number of user increases.

MATLAB coding is done to generate Walsh-Hadmard codes \& later MATLAB code is generated for Optical CDMA system using these hadmard codes for six users.

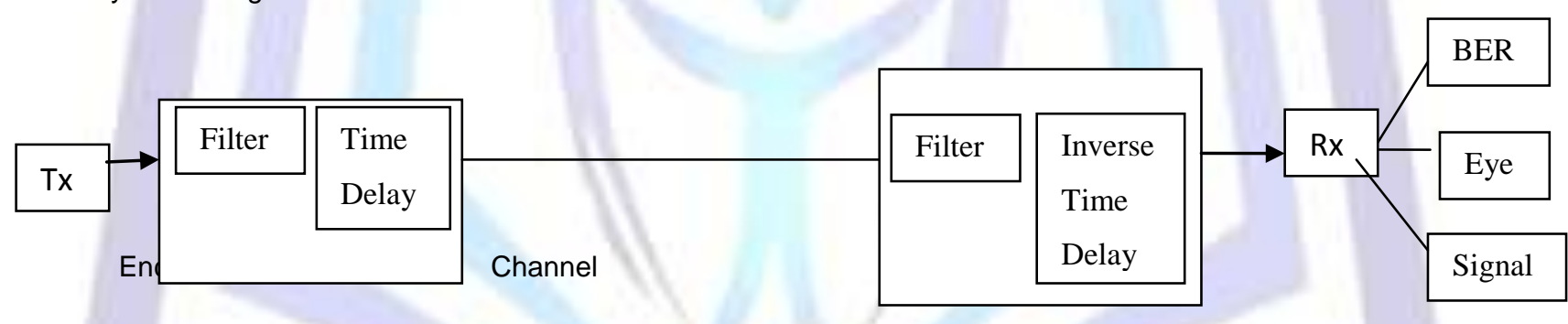

Fig: 1.2. Block Diagram for the implementation of spatial-phase-time codes.

The above Figure 1.2 shows the basic approach to implement the codes. Coding is implemented by First, by varying the space channels and keeping $\mathrm{W}$ and $\mathrm{T}$ constant and latter by converting different wavelengths to different phase shifts. The desired decoded signal can then be separated from all other users' signals by appropriate optical time gating.

\section{III.Simulation setup}

The simulation setup for optical CDMA shown in fig 1.3 implemented phase coding by constructing a matched decoder pair from the hadamard 16 code set. For the demonstration purposes we use 4 equally spaced phase locked laser line within $40 \mathrm{GHz}$ tunable window as frequency slots.

Here a different approach to implement transmitters is adopted. Within a single galaxy it includes

- A carrier wave laser module, LASER CW

- A PRBS,data source module to generate a pseudo-random it sequence

- A coder module,NRZ coder

- An external Mc-Zehnder modulator module, Modulator Diff MZ_DSM

- An electrical DC source, required for external Mc-Zehnder modulator module.

- A module to add the logical channel to the output signal

The very first module inside the setup is the transmitter, which fires first. Whereas inside transmitter PRBS data source fires first. It generates a sequence of single 1's \& 0's at a sample rate of one sample per bit. Hence its output is coded in to multiple samples per bit using NRZ coder, which produces an electrical signal suitable for driving modulator. This module performs coding, slew-rate-limiting, transition shaping, random and deterministic jitter addition, noise addition 
and drive-level setting. For applied NRZ coding, in electrical domain, each it is converted in to number of samples equal to Sample rate/Bit rate.

The band limited electrical sampled signal from the output of coder_Driver_OOK module drives the modulator DiffMZ_DSM module, which modulates the light from the Laser CW module to generate an externally modulated optical pulse sequence. The Coding is implemented. First, the space channels are varied by providing different delay to each transmitter, having different frequency spacing's. The output for all the transmitters is combined and again On/Off (AM) modulated

we have utilized the advantage of discrete frequency lines and small tunable window and then phase encoding technique is employed over each slot using a coder based upon hadamard codes and ultra high resolution optical demultiplexer. The WDM-DEMUX demultiplexes 6 WDM channels. It's galaxy which contains 6 optical band pass filters, an optical attenuator and a logical fork to split the signal having the physical parameters like Bessel's transfer function and stop bandwidth 8 times bit rate. In our OCDMA system every users uses all the lines but with different phase encoding. We can say, this encoder encodes the data both in wavelength and in time domain. Hence allows the much larger number of total users than in the static WDM case. Application of these codes results in temporal pattern which has ' 0 ' optical pulse at the instant in time, where the initial pulse would have had its maximum power.

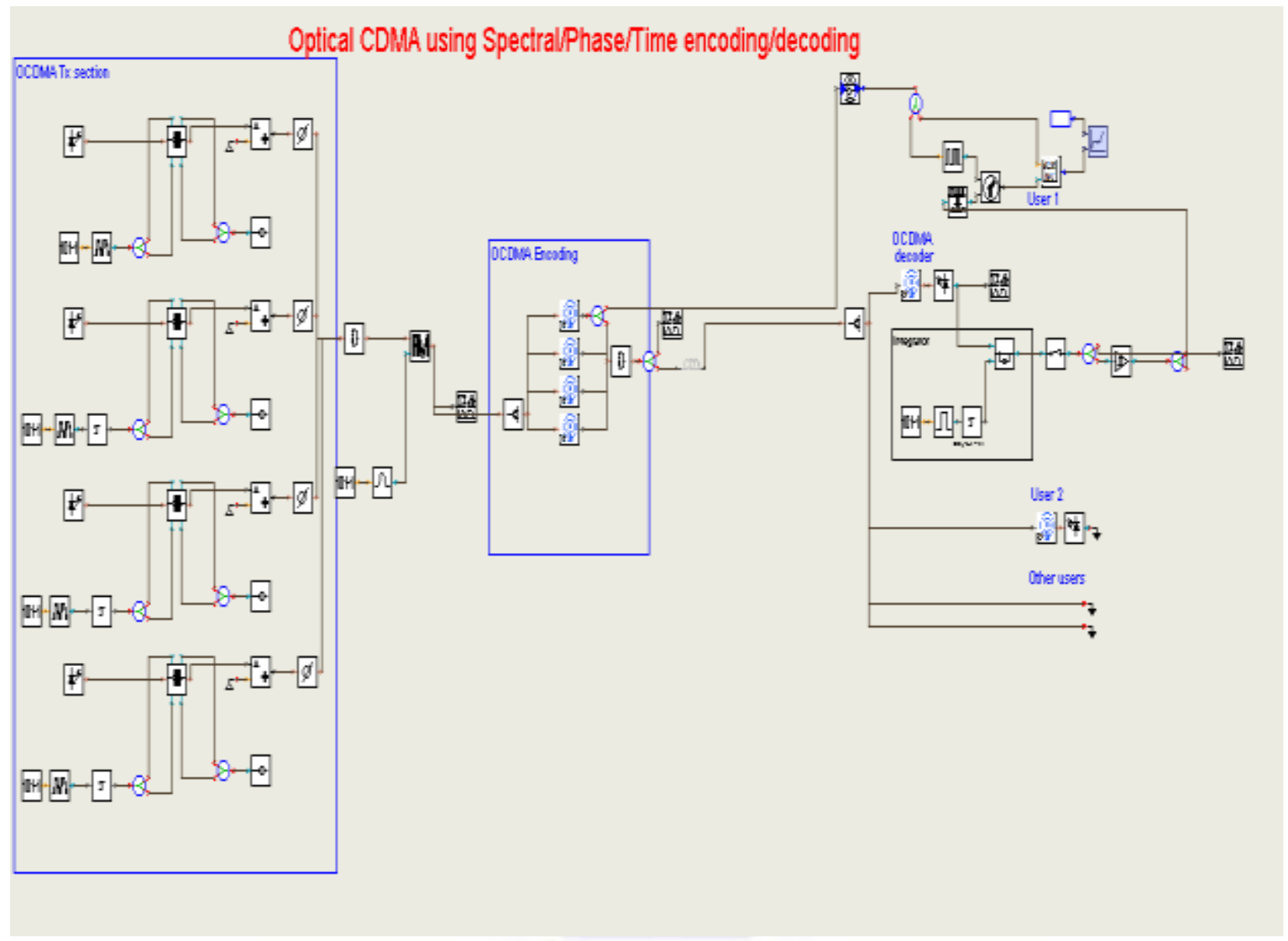

Fig: 1.3 Simulation setup of OCDMA system based on spectral/phase/time encoding/decoding

At the encoder, a WDM Demux demultiplexes the WDM channels \& then delay signal module simulates a propagation delay of optical signal by applying a time shift of the signal waveform. After this a WDM Mux module multiplexes WDM channels which further passes these to modulator where again delay signal module is introduced.

Decoding is accomplished by using optical power splitter which equally splits the incoming signal on each output port and using matched complementary codes at OCDMA decoder, for the binary hadamard codes used here, these codes having its own compliment and consequently both encoder and decoder are identical. Decoder is nothing just inverse of encoder, it consists the same components filter and time delay. We can say, decoder is taken by inverting all the values of encoder as depicted in Fig 1.4 \& 1.5 below. In decoder the delay module simulates a propagation delay of optical signal by applying a time shift of the signal waveform (sampled signals).For optical signals; a delay-induced phase shift is simulated for relative signal frequency. 


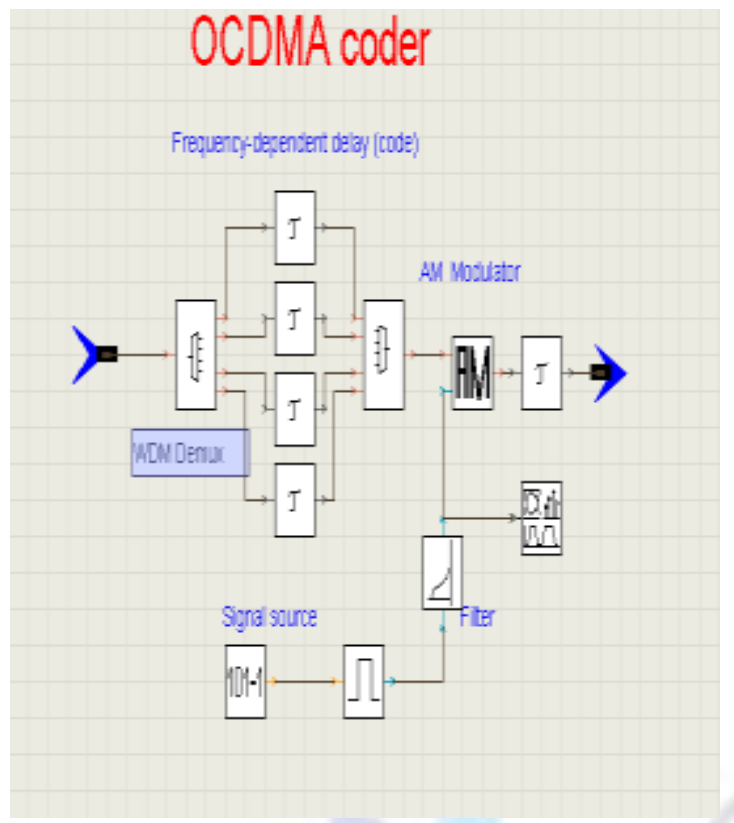

Fig: 1.4 OCDMA Coder

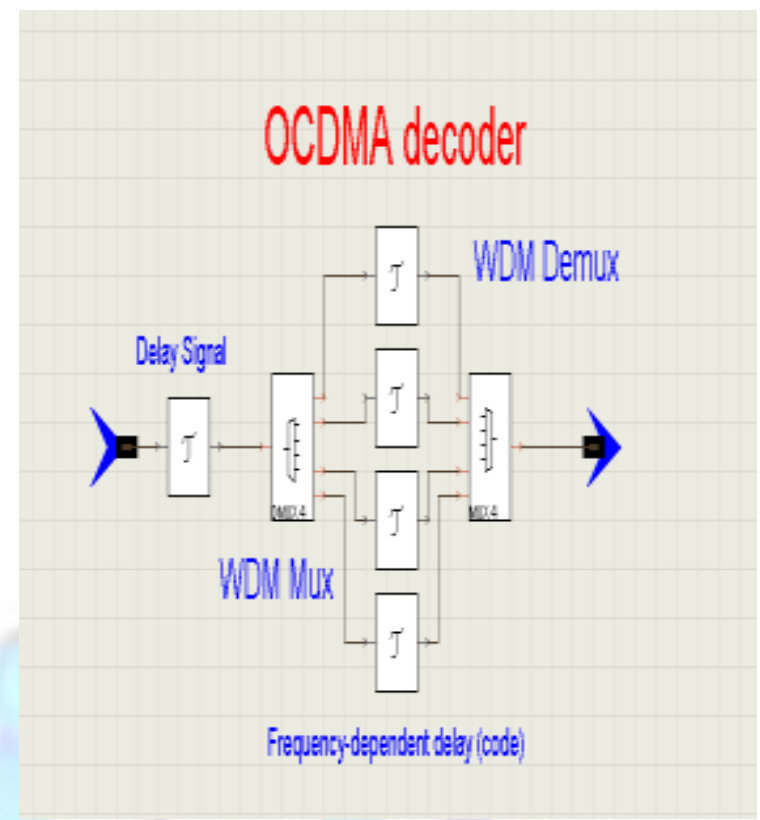

Fig: 1.5 OCDMA Decoder

Output of the six coders is multiplexed into a fiber with a decoder capable of changing its code to match any one of the six transmitters. Output of the decoder is time gated to select the proper sender and reject the MUI from the other five and in turn fed to an optical-electrical receiver. The desired decoded signal can be separated from all other users' signals by optical time gating.

\section{IV.RESULTS AND DISCUSSIONS}

We have demonstrated a novel OCDMA coding and decoding method based on phase coding of tightly spaced phaselocked laser lines that is compatible with conventional WDM networking. This OCDMA system has been demonstrated to accommodate six simultaneous users at $5 \mathrm{~Gb} / \mathrm{s}$ with BER $\leq 10-9$. Further simulation aids in the selection of optimal code sets and provides guidance for appropriate modulation formats to optimize system performance in the presence of MUI and transmission impairments. Simulation results shows that the impairments due to nonlinear optical processes are suppressed in part due to reduced peak power, since coding spreads optical power across the bit period, which is much larger than the MLL/PLL pulse duration.

Using the simulation setup, the values of input signals, wavelength spectrum, eye diagrams and received signals are measured. The received signal is measured at the receiver end. The measurement components used are signal analyzer for the wavelength spectrum, eye diagram analyzer for an eye diagram and signal plotter for the input signal and the received signal. Eye diagram is measured at receiver end for different choice of modulation formats.

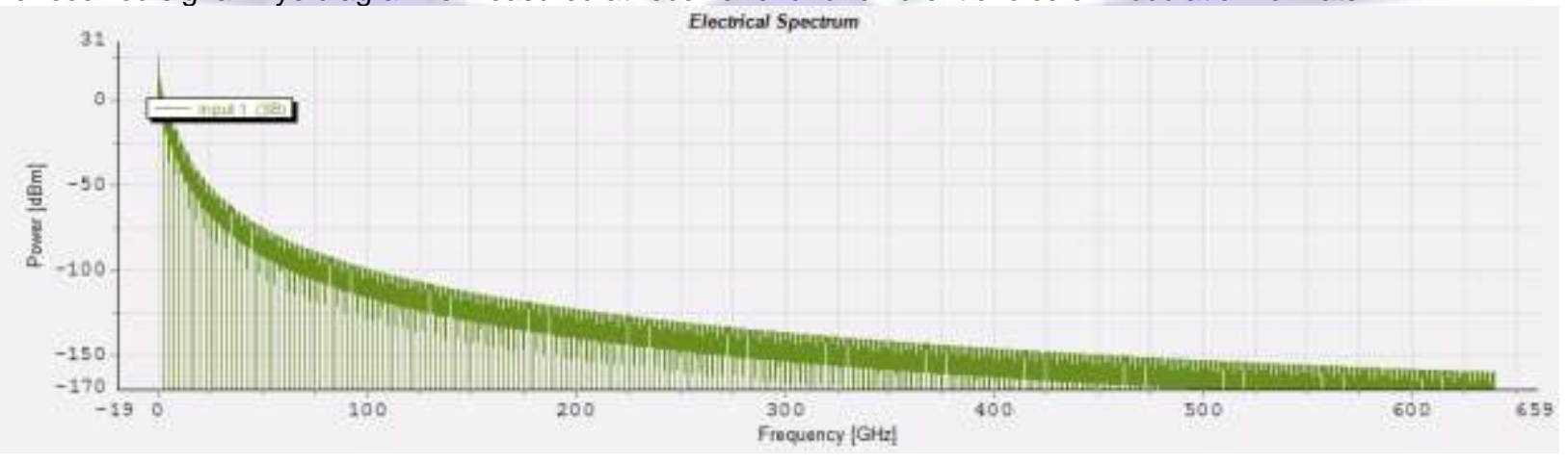

Fig: 1.6 Electrical spectrums at the transmitter 
The phase locked comb, shown in Fig 1.6, is generated by mode locked lasers (MLL). The lines are longitudinal modes of the MLL, having spacing equal to the pulse repetition rate; in our case it is $5 \mathrm{GHz}$.

The transmitted \& decoded data for user1 is shown below in fig 1.7 \& 1.8 ; using $5 \mathrm{GHz}$ frequency slots to code $5 \mathrm{~Gb} / \mathrm{s}$ data. It clearly shows that frequency slots are adequately wide to carry this data rate. Similar waveforms are obtained for other users.

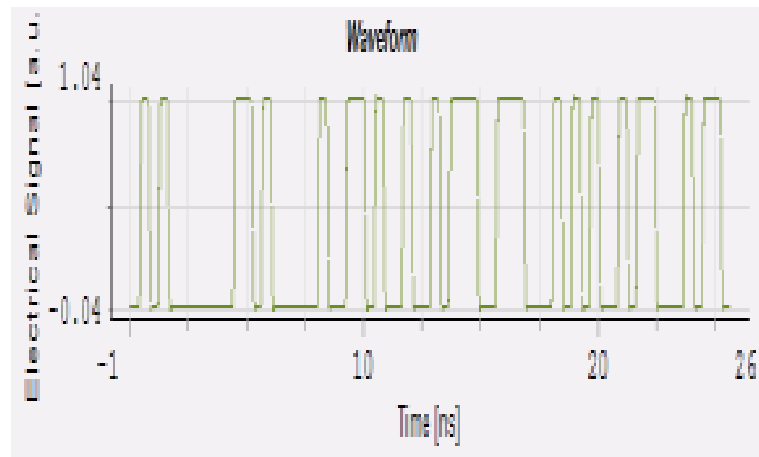

Fig 1.7 Transmitted waveform for channel 1

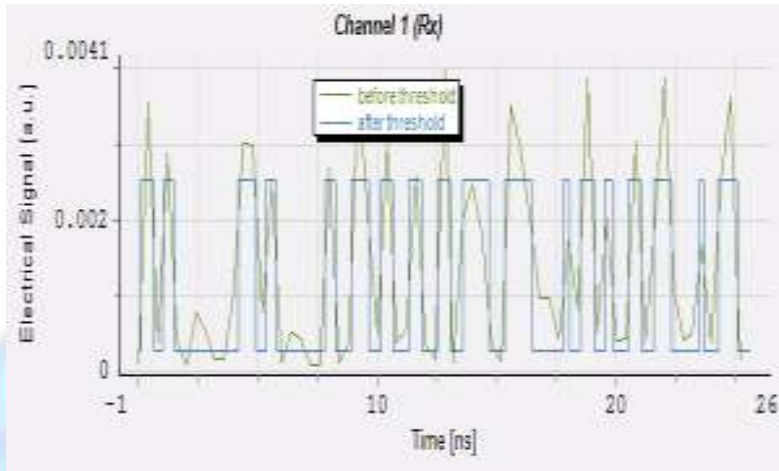

Fig 1.8 Detected waveform for channel 1

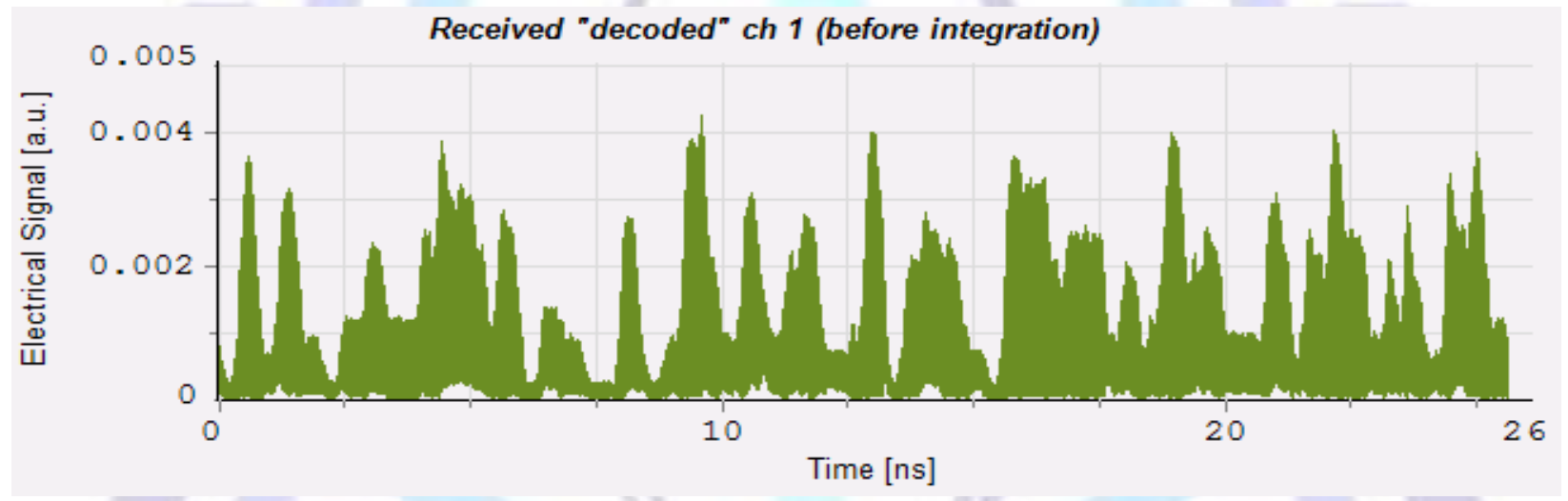

Fig 1.9 Detected waveform for channel 1(before integration)

Fig 1.9 shows received waveform for channel 1 prior to integrator. This is a result of simulation of decoded user (channel1) in the presence of five other users with different codes. In this the optical power associated with the interfering users is present but it is displaced in time domain from the decoded data, which alone gives a clear eye. After this the detected electrical signal passes through an integrator, which is a lossy integrator for electrical signals, where a portion of previous output is added to new input to give an output. If upper limit is not equal to lower limit, the integrator will saturate at the upper limit \& lower limit, or will wrap-around (when beyond these limits).

Fig below shows the eye diagram at the receiver side for the OCDMA system using On/Off key (AM) modulation for 75 $\mathrm{Km}$ fiber length.

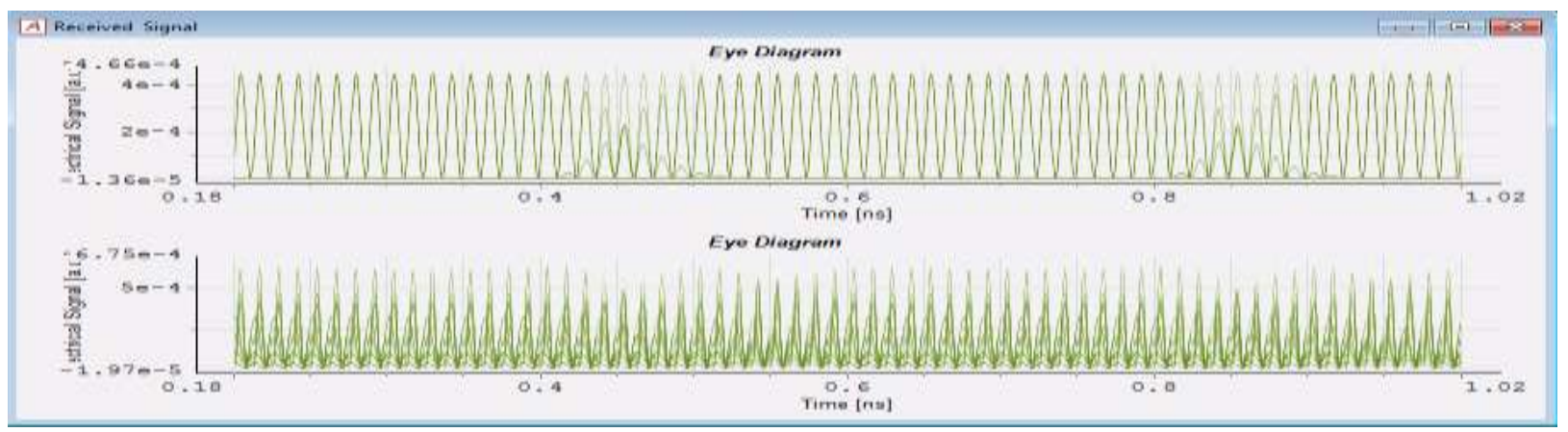

Fig 1.10 Eye-diagram for on/off key modulation 
Hence initial measurements have shown that it is quite possible to code and decode a pulse stream \& results obtained have indicated that frequency slots are adequately wide to carry this data rate. Our simulations show that the open eye in optical time gating window corresponds to a BER well below acceptable value (i.e. BER $\leq 10-9$ ). Because of electrical processing (integration) only error-counting can be used to estimate the BER.If we avoid the use of this the laser line width will reduce and time window will increase. Hence suitable changes are made in the setup to compute BER value w.r.t Qfactor value for different length of fibers i.e from 75 to $95 \mathrm{Kms} \&$ are presented below

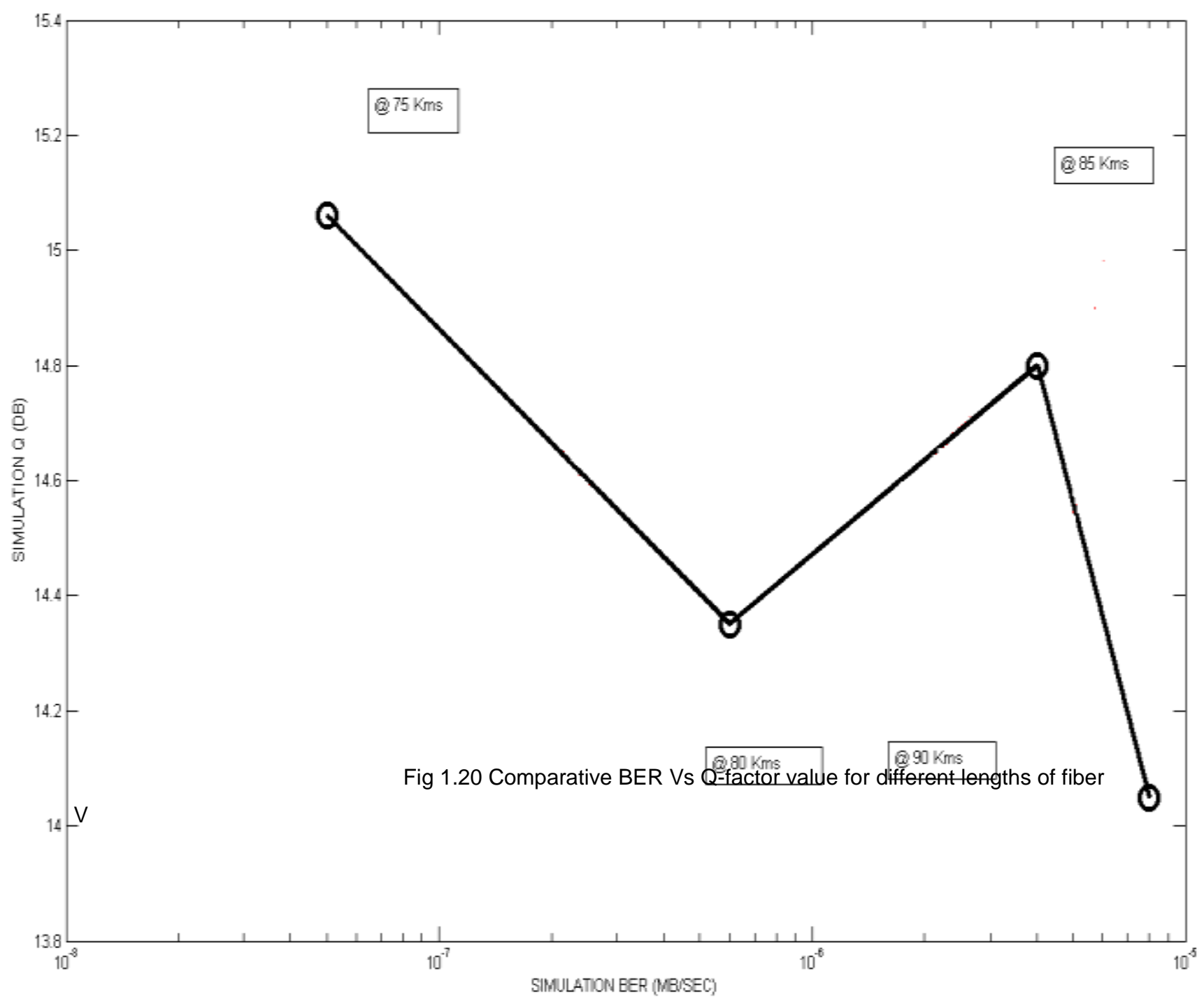

\section{Conclusion}

We have demonstrated a six-user OCDMA system operating at $5 \mathrm{~Gb} / \mathrm{s}$ with a low-power requirement per user, which shows potential for scaling of OCDMA at multigigabit-per-second data rates to significantly higher user counts. The coding and decoding method used is based on 3-D coding of tightly spaced phase-locked laser lines that is compatible with conventional WDM networking. Six channels (5 Gbps per channel) are encoded on 6 frequency components within a 40 $\mathrm{GHz}$ tunable window frequency slots. The narrower spectral extent of the coded signal also limits the effects of transmission impairments such as dispersion. This paper presents a novel non-uniform encoding scheme or synchronous O-CDMA systems using ultra-short coherent optical pulses of arbitrary power spectra. Simulation results demonstrate significant improvement in terms of the orthogonality of the interfering waveforms among users. A BER reduction is obtained for a system implementing phase codes. Simulation indicates that $50 \%$ spectral efficiency can be achieved with these coders with eight simultaneous users at $5 \mathrm{~Gb} / \mathrm{s}$, if duo binary modulation is used to limit spectral spreading. Since the C-band accommodates many such windows, this approach, applied to each window, can support more than 100 simultaneous users. 


\section{REFERENCES:}

[1]. A. J. Mendez, R. M. Gagliardi, V. J. Hernandez, C. V. Bennett, and W. J. Lennon, "Design and performance analysis of wavelength/time (W/T) matrix codes for optical CDMA," IEEE Journal of Light wave Technology, vol. 21, pp. 524-2533, Nov. 2003.

[2] A. J. Mendez, R. M. Gagliardi, V. J. Hernandez, C. V. Bennet and W. J. Lennon, "High-performance optical CDMA system based on 2-D optical Orthogonal codes," IEEE Journal of Light wave Technology, vol. 22, pp. 2409- 2419, Nov. 2004.

[3] A. J. Mendez, R. M. Gagliardi, H. X. C. Feng, J. P. Heritage, and J. M. Morookian, "Strategies for realizing optical CDMA for dense, high-speed, long span, optical network applications," IEEE Journal of Lightwave Technology, vol. 18, pp. 1685-1697, Dec. 2000.

[4] Xiaoli Sun and Fredric. M Davidson, "Photon Counting with Silicon Avalanche Photodiodes", Journal of Lightwave Technology, Vol 10, No 8, August 1992.

[5] Andrew Stok and Edward H. Sargent, "System Performance Comparison of Optical CDMA and WDMA in a Broadcast Local Area Network", IEEE Communication Letters, Vol 6, No. 9, pp 409-411, September 2002.

[6] J.A. Salehi, "Code division multiple access techniques in optical fiber networks part 1: Fundamental principles," IEEE Transactions on Communication, Vol. 37, No. 8, pp. 824-833, August 1989.

[7] E.S. Shivaleela, A. Selvarajan, T. Srinivas, Two-dimensional optical orthogonal codes for fiber-optic coma networks, J. Lightwave Technol. 23 (2) (2005) 647-654.

[8] E.S. Shivaleela, K.N. Sivarajan, A. Selvarajan, Design of a new family of two-dimensional codes for fiber-optic cdma networks, J. Light wave Technol. 16 (4) (1998) 501-508.

[9] R.S. Kaler, Ajay K.Sharma \& T.S. Kamal, "Comparison of pre-, post- and symmetrical-dispersion compensation schemes for $10 \mathrm{~Gb} / \mathrm{s}$ NRZ links using standard and dispersion compensated fibers," Optics Communications 209 (2002) 107-1231 August 2002.

[10] Z. Jiang, S.-D. Yang, Student Member, IEEE, D. E. Leaird, R. V. Roussev, C. Langrock, M. M. Fejer, and A. M. Weiner, "Four-User, 2.5-Gb/s, Spectrally Coded OCDMA System Demonstration Using Low-Power Nonlinear Processing" Journal of Lightwave Technology, Vol. 23, No. 1, January 2005.

[15] Yixue Du, S. J. B. Yoo, and Zhi Ding, "Non-Uniform Spectral Phase Encoding in Optical CDMA Networks," IEEE Photonics Technology Letters, Vol. 18, No. 23, December 1, 2006.

[16] K.J Horadam "Hadamard Matrices and Their Applications" Princeton University Press 1986. 